\title{
The Rate of Convergence of Fourier Coefficients for Entire Functions of Infinite Order with Application to the Weideman-Cloot Sinh-Mapping for Pseudospectral Computations on an Infinite Interval
}

\author{
JOHN P. BOYD \\ Department of Atmospheric, Ocean \& Space Science and Lahoratory for Scientific Compuation, University of Michigan. \\ 2455 Halward Avenue, Am Arbor, Michigan 48109
}

Received December 7, 1992; revised July 2, 1993

\begin{abstract}
We analytically compute the asymptotic Fourier coefficients for several classes of functions to answer two questions. The numerical question is to explain the success of the Weideman-Cloot algorithm for solving differential equations on an infinite interval. Their method combines fourier expansion with a change-of-coordinate using the hyperbolic sine function. The sinh-mapping transforms a simple function like $\exp \left(-z^{2}\right)$ into an entire function of infinite order. This raises the second, analytical question: What is the Fourier rate of convergence for entire functions of an infinite order? The answer is: Sometimes even slower than a geometric series. In this case, the Fourier series converge only on the real axis even when the function $u(z)$ being expanded is free of singularities except at infinity. Earlier analysis ignored stationary point contributions to the asymptotic Fourier coefficients when $u(z)$ had singularities off the real $z$-axis, but we show that sometimes these stationary point terms are more important than residues at the poles of $u(z)$. IC: 1994 Academic Press, Inc.
\end{abstract}

\section{INTRODUCTION}

There are many ways to apply spectral methods to an infinite interval $[1-17]$. Weideman and Cloot $[6,7]$ proposed a new two-part strategy. First, map the physical coordinate $z \in[-\infty, \infty]$ to the new computational coordinate $\zeta$ via

$$
z=\sinh (L \zeta)
$$

where $L$ is a user-choosable constant, the "map parameter." Second, expand the solution as a Fourier series in $\breve{:}$

$$
u(z(\zeta))=a_{0}+\sum_{n=1}^{\infty} a_{n} \cos (n \zeta)+\sum_{n=1}^{\infty} b_{n} \sin (n \zeta)
$$

and compute the coefficients by the usual Fourier pseudospectral method $[1,6]$.
The Weideman-Cloot algorithm is both simple and efficient. Unlike alternative infinite interval basis sets like Hermite functions, Fourier series can be manipulated by the fast Fourier transform. More important, Weideman and Cloot show that their scheme is even more effective than the carlier mapping-and-Fourier methods of Grosch and Orszag $[8]$ and Boyd $[2,4,5]$.

To understand this success, we need to answer four (apparent) criticisms of their algorithm. First, their mapping is a form of domain truncation; that is, the infinite interval is approximated by the large but finite interval $z \in[-D, D]$ where $D=\sinh (L \pi)$. This implies a "domain truncation error,"

$$
E_{D} \sim O[|u( \pm D)|] .
$$

This seems a drawback because this particular error is absent from alternatives that compute on the whole infinite interval such as Hermite functions. However, if $u(z)$ decreases exponentially with $z$, as true in most applications, then $E_{D}$ decreases exponentially fast with $D$. Thus, domain truncation is an effective strategy if $D$ is sufficiently large.

The second criticism is that we need to choose the map parameter $L$. However, as stressed in Boyd $[2-4]$, all infinite interval methods either explicitly or implicitly contain a scaling parameter $L$ whose choice is important for efliciency.

The third objection to the Weideman-Cloot algorithm is that $u(z[\zeta])$ is not periodic in $\zeta$. If $u(z[\pi]) \neq u(z[-\pi])$, then the Fourier series will exhibit Gibbs' phenomenon with a maximum pointwise crror of about $9 \%$ of the jump,

$$
E_{\text {Gibbs }}=0.089\{u(z[\pi])-u(z[-\pi])\},
$$

independent of the truncation of the Fourier series. 
Boyd [5] pointed out, however, that

$$
E_{\text {Gibbs }}<0.18 E_{D}
$$

that is, the "Gibbs error" is always small in comparison to the domain truncation error $E_{D}$. Furthermore, maximum error is always located within a small neighborhood of the endpoints where the numerical solution is corrupted by the truncation of the domain (Fig. 1). Thus, it is always safe to combine a Fourier series with domain truncation, with or without a mapping.

The fourth objection is far more subtle and serious than the first three. Because it employs exponential functions, the Weideman-Cloot map (1.1) makes the transformed functions very strongly singular at $\zeta=\infty$ in comparison to the mappings of Grosch and Orszag [7] and Boyd [2, 4, 5], which use only algebraic functions of $\zeta$. This implies that for the "steepest descent" or "stationary point" contributions to the asymptotic spectral coefficients, the Weideman-Cloot mapping is always inferior-in the asymptotic limit $N \Rightarrow \infty$, where $N$ is the truncation of the Fourier series--to the mappings of Grosch, Orszag, and Boyd. The goal of this work is to resolve this paradox to explain why the WeidemanCloot algorithm, although inferior in the limit, is so effective for moderate $N$.

The resolution of the paradox is that the WeidemanCloot method is superior for small $N$ although inferior for
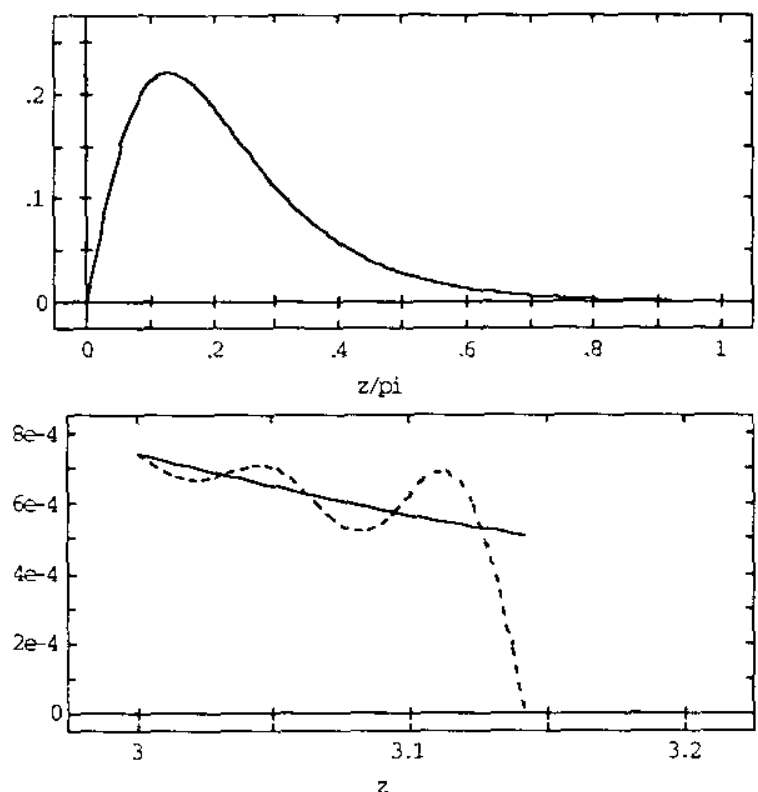

FIG. 1. A schematic illustrating the convergence of the Fourier series for a function which is exponentially small at the ends of the interval. Upper panel: The sum of the first 100 sine terms for the non-periodic function $u(z)=z \operatorname{sech}(3 z)$. Gibbs' phenomenon in invisible on the scale of this graph. Lower panel: Some as the top except for the restricted interval $z \in[3 \pi]$. Note that the $y$-scale is more than 300 times smaller in the lower figure. The relative error of the Fourier series (dashed) near $z=\pi$ is large compared to $f(\pi)$, but the absolute error is tiny because $f(z)$ (solid) is so small near the endpoints. large $N$. The crucial point is that the "crossover point" where the Weideman-Cloot algorithm loses its superiority is very large $N$. For such huge truncation, the error of both schemes is many orders of magnitude smaller than even quadruple precision. For reasonable $N$, the WeidemanCloot mapping is better for entire functions.

For non-entire functions, that is, functions which have singularities, a finite distance from the real axis, the asymptotic error depends on additional terms besides the stationary point contributions. Because of these "singularity terms," for non-entire functions, the Weideman-Cloot mapping is superior for all $N$.

The reason for its superiority is that infinite interval methods must balance two conflicting needs. Large map parameter $L$ reduces the domain truncation error (good) but spreads the points of the computational grid near the origin, decreasing resolution (bad) as shown in Fig. 2. For any mapping and any truncation $N$, the optimum map parameter $L$ is a compromise between the need to minimize domain truncation error while simultaneously minimizing resolution errors for small $z$.

The virtue of the Weideman-Cloot algorithm is that it is very good at compromise. Because $D(=\sinh (L \pi))$ grows exponentially with $L$, only a slight increase in $L$ will greatly increase the domain size $D$. Recalling that $E_{D}$ decreases
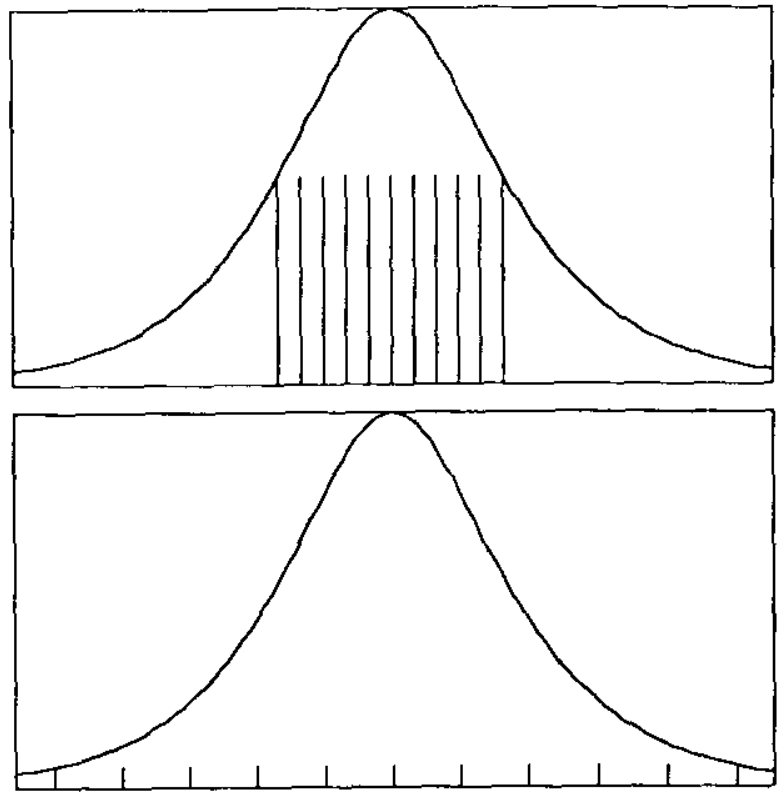

FIG. 2. Schematic illustrating the trade-offs in choosing a map parameter $L$ (or domain size $D$ ) to be large or small. The vertical line segments mark the position of each grid point through their own position on the $z$-axis; the length of each segment shows the domain truncation error $E_{D}$ for each grid. Thus, the outermost segments, at $z= \pm D$, just touch the graph of $u(z)$. Top: Small $L$. The grid points are close, so resolution of the peak is very good, but the cost is that the domain error is large Bottom: Large $L$. The domain truncation error is much smaller because the computational domain is much larger, but the resolution of interior features is poor because the grid points are now much farther apart. 
exponentially fast with $D$, we see that $E_{D}$ decreases as an exponential-of-an-exponential of $L$. Thus, it is always sufficient to use $L \sim O(1)$, which gives excellent resolution for small $z$.

We show below that the Weideman-Cloot algorithm achieves what we shall dub "quasi-geometric convergence": If $E_{T}(N)$ is the total error as a function of the Fourier truncation $N$, then

$$
\log \left(E_{T}\right) \sim-p N / \log (N)
$$

for some eonstant $p$.

To prove this and to show that (1.6) really is excellent in comparison to its competitors, we shall use the two-part methodology of Boyd [2-4]. First, choose model functions, either entire functions or functions with off-axis poles or branch points, which are representative of broad classes of functions. Second, apply the method of steepest descent and the calculus of residues, as pioneered by Elliott [18-20] and Miller [21], to analytically calculate the Fourier coefficients of the models in the asymptotic limit $n \Rightarrow \infty$.

In Section 2, we briefly review different rates of convergence for spectral series. The following section describes the mechanics of the method of steepest descent.

\section{A REVIEW OF RATES-OF-CONVERGENCE THEORY FOR FOURIER SERIES}

Fourier convergence theory can be summed up in an echo of a Biblical proverb: By their singularities shall we know them. What this means is that asymptotically, as $n \Rightarrow \infty$, the coefficients of the Fourier series of a function are the sum of contributions from each singularity of $u(z)$ [1]. The singularities fall into four classes, each implying a qualitatively different rate of convergence as illustrated schematically in Fig. 3. The classes are the following:

(i) Poles, branch points, and discontinuities of $u(z)$ or a derivative on the real axis. If present, these singularities imply an algebraic rate of convergence, such as $a_{n}=1 / n^{2}$. (Example: $u=z^{1 / 2}$.)

(ii) Singularities which have an infinite number of bounded derivatives for all real $z$, but nevertheless destroy the analyticity of $u(z)$ for some real $z$. Mathematically, $u(z)$ is $C^{\infty}$ but not $C^{\Omega}$. These imply subgeometric convergence, such as $a_{n}=\exp \left(-n^{2 / 3}\right)$. (Example: $u=\exp \left(-1 / z^{2}\right)$.)

(iii) Poles and branch points a finite distance $s_{j}$ from the real axis. These imply geometric convergence, such as $a_{n}=\exp (-n / 2)$. (Example: $u=1 /(2+\cos (z))$.)

(iv) Singularities at $z=\infty$. If these are the only poles or branch points of $u(z)$, then the function is "integral" or "entire" and the rate of convergence is supergeometric, such as $a_{n}=\exp ((1 / 2)[1-n \log (n)])$. (Example: $u=$ $\exp (-\cos (z))$.)
In summary, the complete asymptotic approximation to the Fourier coefficients for $n \gg 1$ is of the form

$$
\begin{aligned}
a_{n} \sim \sum_{j=1}^{n_{\text {real }}} \alpha_{j} n^{-q_{j}}+\sum^{n_{C} \infty} \beta_{j} \exp \left(-p_{j} n^{r_{j}}\right)+\sum_{j=1}^{n_{\text {complex }}} \gamma_{j} \exp \left(-s_{j} n\right) \\
\quad+\sum_{j=1}^{n_{s}} \delta_{j} \exp \left(-t_{j} n / \log (n)\right)
\end{aligned}
$$

where some of these sums may be absent.

The first summation is over those singularities of $u(z)$ on the real $z$-axis, including discontinuities induced by a lack of periodicity. Such poles and branch points contribute terms which are algebraic functions of $n$, usually inverse powers of $n$, perhaps modified by logarithmic factors, omitted for visual simplicity, from (2.1). The power of $n, q_{j}$, is controlled by the type of singularity. Thus, a discontinuity generates a term decreasing as $O(1 / n)$, a discontinuity in the first derivative generates one proportional to $O\left(1 / n^{2}\right)$, and $z^{1 / 2}$ creates a contribution which is $O\left(n^{-3 / 2}\right)$. In the limit $n \Rightarrow \infty$, the sum over real axis singularities will dominate the other sums and the series will have algebraic convergence.

The second sum is the effects of singularities of $u(z)$ which are on the real axis, but are $C^{\infty}$, that is, have bounded derivatives of all orders. The function $\exp \left(-1 / z^{2}\right)$, for example, has an essential singularity at the origin and thus is not analytic at $z=0$. However, all its derivatives are bounded at the origin (and, in fact, are all zero.) If such $C^{\infty}$ singularities are present (but no singularities of the first class such that $r$ th derivative is unbounded for some finite $r$ ), then the series will have subgeometric convergence. Typically, the dominant term is an exponential of $n$ raised to a power

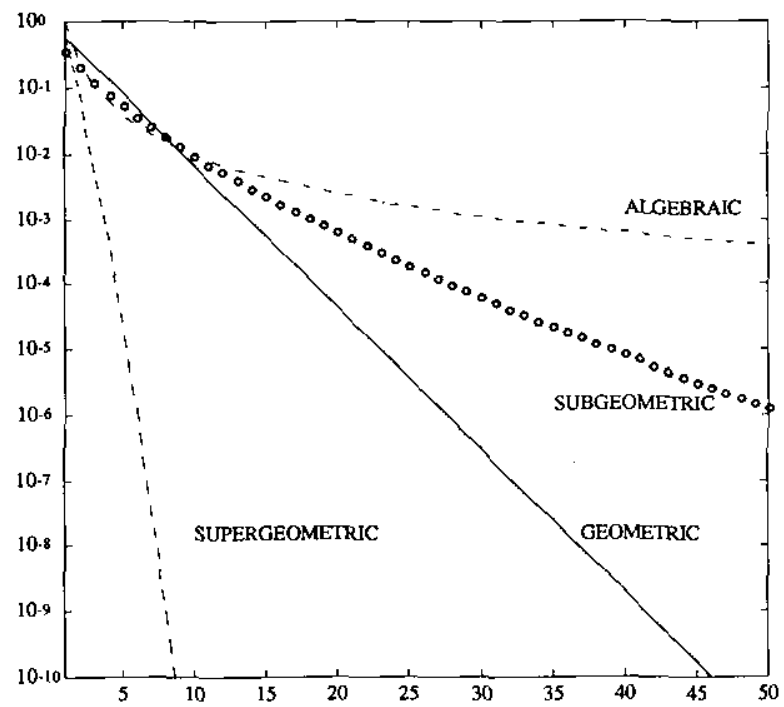

FIG. 3. A schematic illustrating four different species of convergence for spectral series. The logarithm of the total error is plotted versus the Fourier truncation, $N$. 
smaller than one. Again, this exponential may be multiplied by more slowly varying factors which have been omitted from (3.1).

The third sum represents poles and branch points which are located a finite distance from the real $z$-axis. The exponential $\exp \left(-\mu_{j} n\right)$ can be written as $\left(\exp \left(\mu_{j}\right)\right)^{-n}$, so the terms decrease like the terms of a geometric series, hence the name "geometric convergence." The constants $\mu_{j}$ are simply the distance of the $j$ th pole or branch point from the real axis, that is, the absolute value of the imaginary part of the location of the singularity. The smallest $\mu_{j}$ is $\mu$, the "asymptotic rate of convergence" as defined in [1]. It is obviously important whether $\mu$ is very small or very large, and the goal of the steepest descent analysis is to determine $\mu$ and related parameters.

A geometrically converging series converges throughout the whole strip in the complex $z$-plane such that $|\operatorname{Im}(z)|<\mu$. To put it another way, the Fourier series converges within the largest strip, parallel to the real axis, which has a pole or branch point of $u(z)$ on one of the lines, $\operatorname{Im}(z)= \pm \mu$, which bounds the strip.

The fourth sum is over terms which are associated with singularities of $u(z)$ at infinity. These terms are the contributions of so-called "stationary points" when the integrals that define the Fourier coefficients are asymptotically evaluated by the method of steepest descent, so we will call these the "stationary point" terms. These dominate, giving supergeometric convergence, only when $u(z)$ is free of singularities except at infinity. The form of the terms may be different from that shown in (2.1), but always it is an exponential with an argument which increases faster-thanlinearly with $n$. As for the other sums, factors varying more slowly than the exponentials have been omitted from (2.1), as have factors which oscillate with $n$.

To understand the stationary point contributions, it is sufficient to use "entire" or "integral" functions, that is, those for which $u(z)$ is free of poles or other singularities except at infinity. Entire functions can be classified by "order," where $\rho$ is the smallest number for which

$$
|u(z)| \leqslant[\text { const }] \exp \left(M|z|^{\rho}\right)
$$

for all $|z|$. When $1<\rho<\infty$, the steepest descent methods shows that

$$
a_{n} \sim O\left(\exp \left[-t n^{\rho /(\rho-1)}\right]\right) .
$$

However, a change of coordinates drastically alters the situation.

For example, a Chebyshev polynomial series in $x$ is really a Fourier cosine series in the variable $z$ where $x \equiv \cos (z)$. Making this substitution, the Chebyshev series for $\exp (x)$ becomes the Fourier series for $u(z)=\exp (\cos (z))$. Although $\exp (x)$ is an entire function of first order in $x, \exp (\cos (z))$ is an entire function of infinite order in $z$. The result is that the $a_{n}$ decrease as

$$
a_{n} \sim O(\exp [-n \log (n)])
$$

The convergence is still supergeometric, but only by virtue of a factor of $\log (n)$. In contrast, the Fourier series for entire functions (in $z$ ) of finite order $\rho$ replace $\log (n)$ by $n^{1 /(\rho-1)}$. It was observed at least 15 years ago [13] that the Chebyshev coefficients of an entire function of order $\rho$ (in the Chebyshev argument $x$ ) usually decrease as $O(\exp [-(n / \rho) \log (n)]$.

Weideman and Cloot $[6,7]$ neglected the stationary point contributions in their asymptotic analysis for $u(z)$ which have singularities at finite complex $z$. Implicitly, they assumed that the stationary point terms would be supergeometrically converging, too. Although this assumption is harmless for all their examples, we shall show through a counterexample that the stationary point contributions are neither always negligible nor always supergeometrically converging.

\section{THE METHOD OF STEEPEST DESCENT}

In earlier applications $[2,3]$, we asymptotically approximated the usual Fourier coefficient integrals. In this work, we shall adopt a simpler approach of asymptotically approximating the Fourier transform of $u(z)$, thereby avoiding endpoint effects:

$$
a_{n}=\frac{1}{\pi} \int_{-\infty}^{\infty} u(z) e^{i n z} d z
$$

There are two ways to justify (3.1). One is to observe that if $u(z)$ is decaying exponentially fast on the real axis as $|z| \Rightarrow \infty$, then we can extend the integration limits of the usual Fourier coefficient integrals from $\pm \pi$ to $\pm \infty$ with only an exponentially error. (This error in "integrationlimit-extension" is of the order of magnitude of the domain error $E_{D}$, which must be small if the Fourier series is to be useful.)

The second justification is to define the function

$u_{P}(z) \equiv \sum_{m=-\infty}^{\infty} u(z-2 \pi m) \quad$ ("imbrication of $f(z)$ ").

This new function $u_{P}(z)$, which is spatially periodic by construction, is said to be the "imbrication" of the "pattern function" $u(z)$. "Imbrication" is a Latin word meaning "to overlap like a pattern of tiles," which is a good description of how the multiple copies of $u(z)$ sum to give a periodic function. For a rapidly decaying $u(z)$, however, the overlap is almost negligible except at the boundaries, where $u_{P}( \pm \pi) \approx 2 u( \pm \pi)=2 E_{D}$. The Poisson sum theorem [23] 
implies that the Fourier coefficients of $u_{p}(z)$ are given by the Fourier transform of the "pattern function," that is, by (3.1). Thus, we have two ways of interpreting (3.1): (i) as the approximate Fourier coefficients of the non-periodic function $u(z)$ or (ii) as the exact Fourier coefficients of the periodic function $u_{P}(z)$.

The method of steepest descents is to deform the contour of integration into the complex $z$-plane in such a way that it passes through one or more "stationary points" where the integrand rises steeply to a tall, narrow maximum. In the vicinity of the stationary point, the integrand may be locally approximated by a Gaussian function whose integral can be evaluated in closed form. The integral is approximated by the sum of the contributions from each stationary point. If we define a "phase function" $\phi(z ; n)$ for $u(z)$ via

$$
\phi(z ; n) \equiv \ln (u(z))+i n z \Leftrightarrow u(z) e^{i n z}=e^{\phi(z ; n)}
$$

then the stationary points are the roots of $d \phi / d z$. The integral is approximated by

$$
a_{n} \sim \sum_{s} \exp \left[\phi\left(z_{s} ; n\right)\right] \sqrt{2 /-\pi \phi^{\prime \prime}\left(z_{s} ; n\right)},
$$

where the sum runs over all stationary points that lie on the path of integration and where (") denotes the second $z$-derivative.

The imaginary part of $\phi\left(z_{s} ; n\right)$ gives oscillations with $n$ while the square root factor in (3.4) typically adds a multiplier of $n^{-1 / 4}$ or $n^{1 / 2}$ to $a_{n}$. The crucial part of the asymptotic approximation, however, is the real part of $\phi\left(z_{s} ; n\right)$ because this controls the exponential rate of convergence with $n$.

The following theorem, which has not been emphasized in the literature, is very useful in obtaining this crucial factor. Note that for geometric convergence, $a_{n} \sim \exp (-\mu n)$, $\log \left(a_{n}\right) \sim-\mu n \sim \operatorname{Re}\left(\phi\left(z_{s} ; n\right)\right)$. Thus, $\operatorname{Re}[d \phi / d n]=-\mu$, the "asymptotic rate of [geometric] convergence" [1].

THEOREM 1. In the steepest descent approximation to an arbitrary Fourier transform integral (3.1), the derivative (with respect to $n$ ) of the phase function $\phi\left(z_{s}(n) ; n\right)$ in $(3.4)$ is directly proportional to the corresponding stationary point, $z_{s}(n)$,

$$
\frac{d \phi\left(z_{s}(n) ; n\right)}{d n}=i z_{s}(n)
$$

Thus,

$$
\mathfrak{R}\left(\frac{d \phi\left(z_{s}(n) ; n\right)}{d n}\right)=-\mathfrak{I}\left(z_{s}(n)\right)
$$

Proof. The chain rule implies

$$
\frac{d \phi\left(z_{s}(n) ; n\right)}{d n}=\frac{\partial \phi}{\partial z} \frac{d z_{s}}{d n}+i z_{s}
$$

However, the condition that determines the stationary point $z_{s}(n)$ is that it must be one of the roots of $\partial \phi / \partial z$. Thus, the first term in (3.7) must vanish everywhere along the curve $z_{s}(n)$ in the complex $z$-plane, giving (3.5).

This theorem gives another way of distinguishing between subgeometric, geometric, and supergeometric convergence for the stationary point contributions. If $\operatorname{Im}\left(z_{s}\right)$ tends to a constant $\mu$ as $n \Rightarrow \infty$, then the convergence is geometric with asymptotic rate of convergence $\mu$. If the stationary point moves closer to the real $z$-axis as $n$ increases, then the convergence is subgeometric (for the contribution of that stationary point). Similarly, if $\operatorname{Im}\left(z_{s}\right)$ increases with $n$, be it ever so slowly, then the rate of convergence is supergeometric.

The remaining mechanics of steepest descent are best described in terms of specific cases, to which we turn in Section 5 .

\section{RESIDUES AND SINGULARITY CONTRIBUTIONS}

When the contour of integration is deformed from the real axis to the steepest descent path, the deformation will usually, at least for sufficiently large $n$, pass over the location of one or more of the singularities of $u(\zeta)$ in the complex $\zeta$-plane. If so, then the Fourier integral is no longer given solely by the integration along the steepest descent path, but equals the deformed integral plus additive contributions from each of the included singularities. For poles, these contributions are simply the residues at the poles.

For a simple pole at $\zeta=\zeta_{p}$, the residue will be the product of an $n$-independent factor with $\exp \left(i n \zeta_{p}\right)$. Thus, $\mu \equiv \operatorname{Im}\left(\zeta_{p}\right)$ is the asymptotic rate of (geometric) convergence. When there are multiple singularities inside the deformed contour, the one with the smallest $\mu$, that is, the pole or branch point which is closest to the real $\zeta$-axis, will dominate as $n \Rightarrow \infty$.

Since the mapping $z=\sinh (L \zeta)$ is an entire function with no singularities of its own, all the poles and branch points in the $\zeta$-plane are images of similar singularities of $u(z)$ in the $z$-plane. We can visualize the rate of convergence associated with each singularity by plotting the contours of $\mu(\operatorname{Re}(z), \operatorname{Im}(z))$, where

$$
\mu=(1 / L) \operatorname{arcsinh}(z)
$$

Figure 4 shows such a plot for $L=1$; the contours for other $L$ are identical in shape, but the values attached to those contours must be divided by $L$.

We may dub the contours of constant $\mu$ "equiconvergence" contours since a pole or branch point anywhere along the contour $\mu=\mu_{0}$ in the $z$-plane will contribute a term to the asymptotic Fourier coefficients which decays as $\exp \left(-n \mu_{0}\right)$. The bad news is that a singularity on the imaginary axis above $z=i$ will always contribute a term decaying as slowly as $\exp (-n \pi / 2)$, even if the singularity 


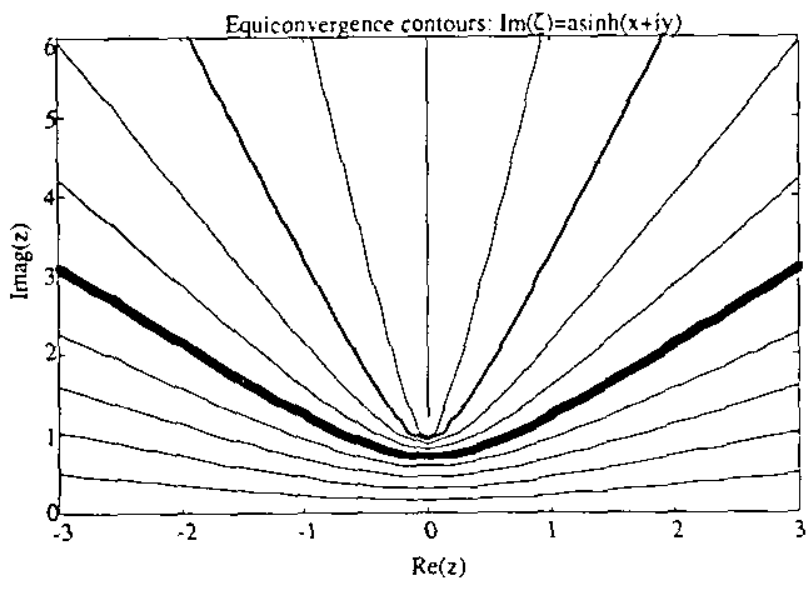

FIG. 4. Equiconvergence contours in $z$-plane, that is, plots of constant values of $\mu$, the imaginary part of $\zeta$, as a function of the real and imaginary parts of $z$. The $\mu$-values of the contours run in even steps of $\pi / 20$ from $\mu=0$ (seal $z$ axis) to $\mu=\pi / 2$ (imaginary $z$ axis above $z=i$ ). The heavy solid line is the contour $\operatorname{Im}(\zeta)=\pi / 4$ in the $z$-plane. For a function with steepest descent contributions decreasing as slowly as $\exp (-n \pi / 4)$, all poles and branch points above this contour in the $z$-plane are irrelevant to the asymptotic Fourier coefficients.

is very, very far up the imaginary $z$-axis. Singularities elsewhere contribute even slower rates of convergence.

The type of singularity affects the contribution only weakly; location is everything. (A proverb in real estate that is equally true for Fourier convergence rates!) A secondorder pole multiplies $\exp (-n \mu)$ by $n$; a logarithm divides this by $n$, and so on. The exponential part of the asymptotic approximation is controlled entirely by the location of the singularity in the $\zeta$-plane.

\section{NUMERICAL EXAMPLES}

\section{The Role of the Map Parameter $L$}

If we define $\alpha(n)$ to be the Fourier coefficients for $L=1$ (for any particular $u(z)$ ), then a simple rescaling of the Fourier transform integral (2.1) shows that

$$
a_{n}(L)=(1 / L) \alpha(n / L)
$$

Thus, it suffices to compute the coefficients for $L=1$, as we shall do in the rest of this section, since we can obtain the coefficients for general $L$ by rescaling.

\section{Gaussian Functions with and without Poles}

Our first example comes in two flavors. The Gaussian

$$
u_{1}(z)=\exp \left(-\frac{1}{2} z^{2}\right)
$$

is an entire function. By dividing this by a factor with complex zeros such as

$$
u_{2}(z)=\frac{\exp \left(-(1 / 2) z^{2}\right)}{z^{2}+b^{2}}
$$

which has simple poles at $z= \pm i b$, we can explore the relative importance of the steepest descent contributions, which are the only terms in the asymptotic approximation to $u_{1}(z)$ versus the contributions of singularities for finite complex $z$.

The transformed version of $u_{1}$, which is illustrated in Fig. 5, and its steepest descent phase function are

$$
u_{1}(z(\zeta))=\exp \left(-\sinh ^{2}(\zeta)\right) \Rightarrow \phi(\zeta ; k)=-\sinh ^{2}(\zeta)+\operatorname{in} \zeta,
$$

where for simplicity $L=1$ as explained above.

The stationary points are the roots of

$$
\frac{d \phi}{d \zeta}=0=-2 \sinh (\zeta) \cosh (\zeta)+i k
$$

By separating (5.5) into real and imaginary parts and trigonometric and hyperbolic identities, the stationary points are, without approximation,

$$
\begin{gathered}
\zeta_{s}= \pm \frac{1}{2} \ln \left(n+\sqrt{n^{2}-1}\right)+i \frac{\pi}{4}+i \mathrm{~m} \frac{\pi}{2} \\
(n>1 ; m=\text { any integer }) .
\end{gathered}
$$
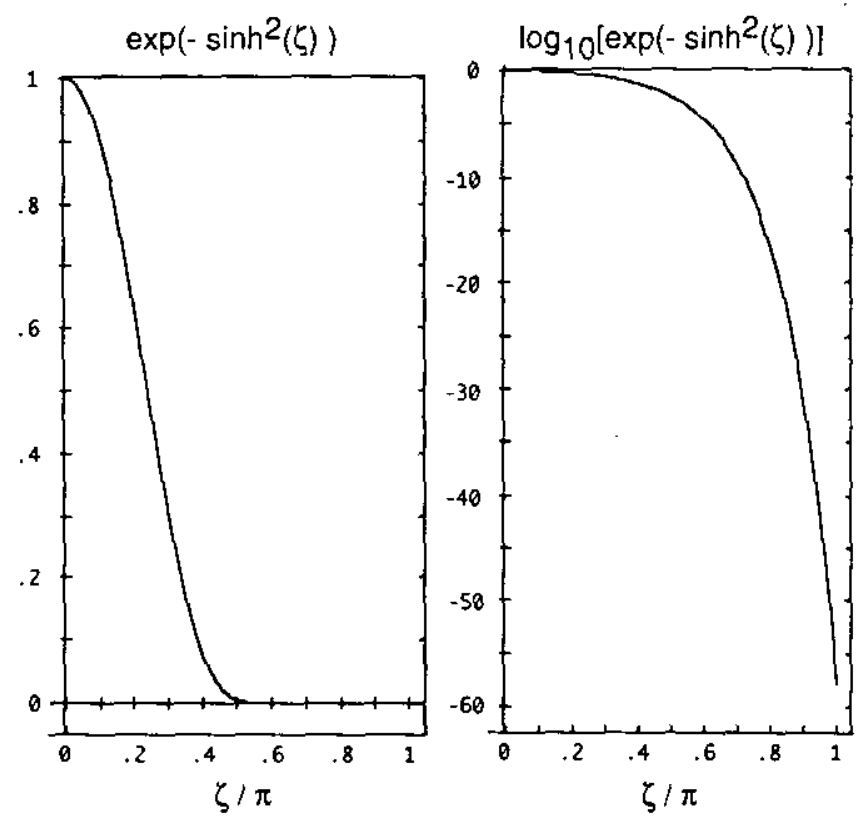

FIG. 5. Two views of the function $u_{1}(\zeta)=\exp \left(-\sinh ^{2}(\zeta)\right)$. Only the half-interval $\zeta \in[0, \pi]$ is shown because the function is symmetric with respect to $\zeta=0$. The left panel uses a linear scale for $u_{1}(\zeta)$ while the right graph shows the $\log _{10}\left(u_{1}(\zeta)\right)$. 
The restriction to $n>1$ is not serious since the steepest descent approximation is accurate only for $n \gg 1$. For negative $n$, the stationary points are the complex conjugate of those in (5.6).

The steepest descent path is shown in Fig. 6, but this does not actually enter the final answer; indeed, we did not compute the deformed integration contour until more than a year after completing a first draft of this paper! An infinite number of stationary points, i.e., $m=0$ and all positive even values of $m$, lie on the deformed contour of integration, which stretches to infinity along the positive imaginary $\zeta$-axis. However, the contributions from the points closest the real axis $(m=0)$ dominate. The contributions of higher $m$ are smaller by $O(\exp [-m n \pi / 2])$. Discarding all but the leading terms and simplifying via trigonometric and hyperbolic identities, we obtain

$$
\begin{aligned}
a_{n} \sim 2 & \sqrt{e / \pi \sqrt{n^{2}-1}} e^{-n \pi / 4} \\
& \times \cos \left\{\frac{n}{2} \ln \left[n+\sqrt{n^{2}-1}\right]-\frac{1}{2} \sqrt{n^{2}-1}-\frac{\pi}{4}\right\} .
\end{aligned}
$$

The "envelope" of the asymptotic approximation may be defined as (5.7) with the cosine factor omitted, i.e.,

$$
e_{n} \sim 2 \sqrt{e / \pi \sqrt{n^{2}-1}} e^{-n \pi / 4} .
$$

We see that, ignoring the oscillatory factor and the $1 / \sqrt{n}$, the Fourier coefficients are decreasing like the terms of a geometric series. That is,

$$
\left|a_{n}\right| \leqslant 1.86\left(\frac{1}{2.19}\right)^{n}=2 \sqrt{e / \pi}\left(e^{-\pi / 4}\right)^{n}
$$

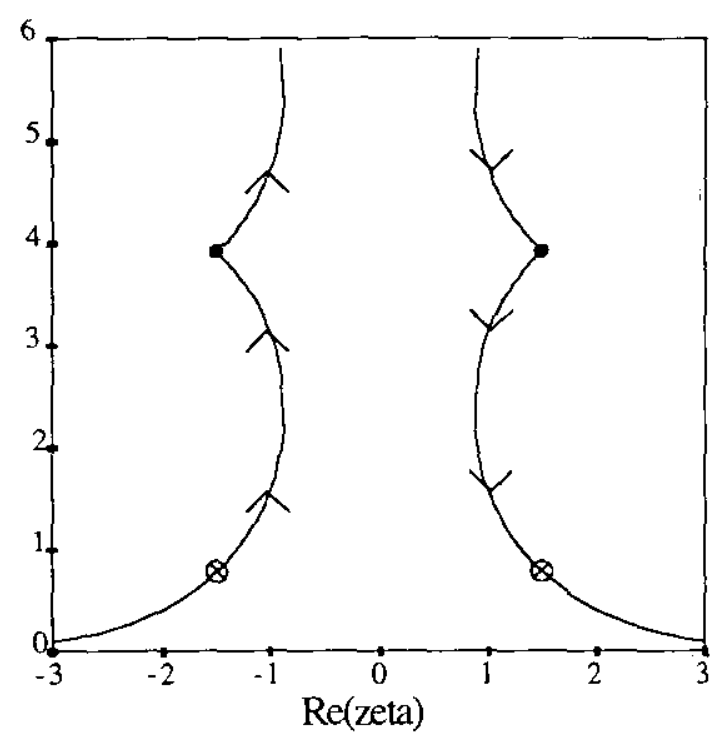

FIG. 6. The steepest descent path in the complex $\zeta$-plane for $u_{1}(z[\zeta])$. The arrows show the direcion of integration. The disks mark the stationary points; the dominant stationary points are marked by the crosses inside the disk. The figure illustrates the path for $n=10$; for other $n$, the imaginary part of the location of each stationary point is unchanged, but the real parts slowly move away from the imaginary axis as $\operatorname{Re}\left(\zeta_{s}\right) \approx \pm\left\{0.35+\ln \left(n^{1 / 2}\right)\right\}$.
Figure 7 and Table I compare the exact Fourier coefficients with the asymptotic approximation. We see that even for small $n$, the asymptotic approximation is quite good.

Multiplying the Gaussian by $1 /\left(b^{2}+z^{2}\right)$ to obtain a singular function modifies the asymptotic Fourier coefficients in two ways. First, the stationary point contributions are multiplied by the value of this factor at the stationary points, i.e., $1 /\left(b^{2}+\sinh ^{2}\left(\zeta_{s}\right)\right)$. Because this multiplier varies slowly with $\zeta$ in comparison with the Gaussian, the algebraic factor does not, except at higher order, alter the location of the stationary points, which are still given by (5.6). For large $n$,

$$
\frac{1}{b^{2}+\sinh ^{2}\left(\zeta_{s}\right)} \sim \frac{2 i}{n}
$$

so the stationary terms are modified only by multiplication by this algebraic (rather than exponential) function of $n$.

Second, the asymptotic Fourier coefficients also include terms generated by the poles. When the path of integration is deformed from the real axis to the contour illustrated in Fig. 6, the path, at least for sufficiently large positive $n$, will be moved across the pole where $\sinh ^{2}\left(\zeta_{p}\right)=i b$. The Fourier integral is then given by the steepest descent term plus the residue at the pole which lies between the old and the new paths. (For negative $n$, the steepest descent path and the enclosed pole are in the lower $\zeta$-plane, the reflections with respect to the real $\zeta$-axis, of the path and pole for $n>0$.)

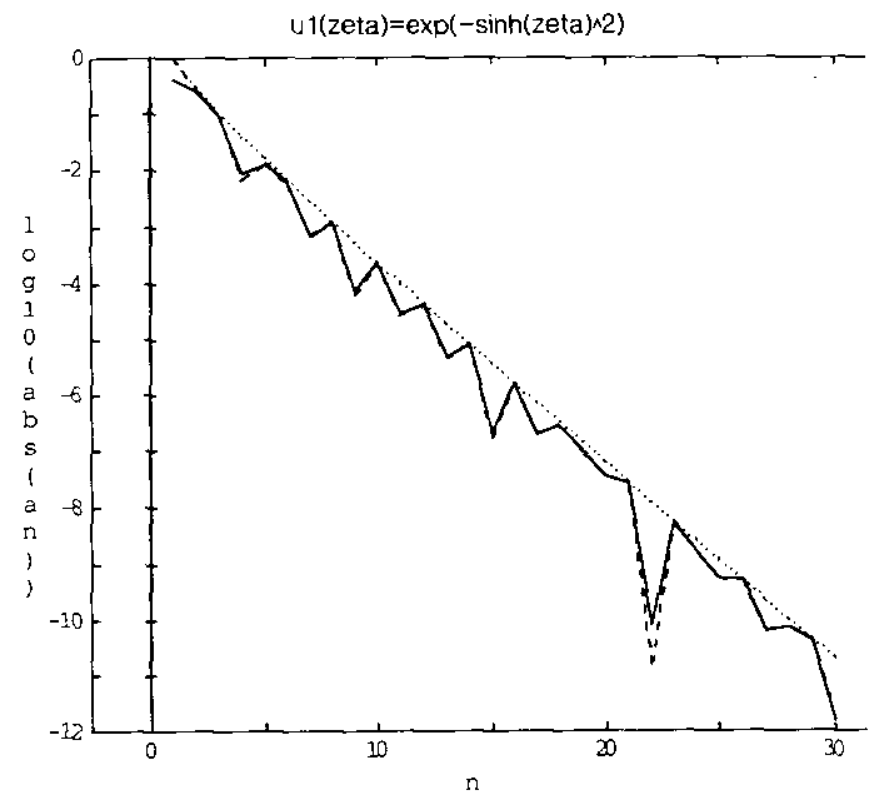

FIG. 7. Solid: logarithm, base 10, of the exact Fourier cosine coefficients for $u_{1}(\zeta)=\exp \left(-\sinh ^{2}(\zeta)\right)$. Dashed: the full asymptotic approximation to the Fourier coefficients, given by (4.7). Dotted: the envelope of the asymptotic Fourier coefficients (4.8). 
TABLE I

Fourier Coefficients, Asymptotic Approximations to the Fourier Coefficients, and the Error in the Asymptotic Approximation for $u_{1}(\zeta)=\exp \left(-\sinh ^{2}(\zeta)\right)$

\begin{tabular}{|c|c|c|c|}
\hline$n$ & $a_{n}$ & Asymptotic & Absolute error \\
\hline 1 & 0.4155 & 1 & -0.585 \\
\hline 2 & 0.2537 & 0.2776 & -0.0239 \\
\hline 3 & 0.09661 & 0.09466 & 0.00196 \\
\hline 4 & $8.660 \mathrm{e}-3$ & $6.743 e-3$ & $1.92 \mathrm{e}-3$ \\
\hline 5 & -0.012832 & $-1.323 e-2$ & $3.98 \mathrm{e}-4$ \\
\hline 6 & $-5.963 \mathrm{e}-3$ & $-5.863 e-3$ & $-1.01 \mathrm{e}-4$ \\
\hline 7 & $6.641 \mathrm{e}-4$ & $7.353 e-4$ & $-7.12 \mathrm{e}-5$ \\
\hline 8 & $1.233 \mathrm{e}-3$ & $1.232 \mathrm{e}-3$ & $4.18 \mathrm{e}-7$ \\
\hline 9 & $7.285 \mathrm{e}-5$ & $6.277 e-5$ & $1.01 \mathrm{e}-5$ \\
\hline 10 & $-2.226 \mathrm{e}-4$ & $-2.235 \mathrm{e}-4$ & $9.09 \mathrm{e}-7$ \\
\hline 11 & $-2.791 \mathrm{e}-5$ & $-2.643 c-5$ & $-1.48 \mathrm{e}-6$ \\
\hline 12 & $4.163 e-5$ & $4.181 \mathrm{e}-5$ & $-1.73 \mathrm{e}-7$ \\
\hline 13 & $4.674 e-6$ & $4.433 e-6$ & $2.41 \mathrm{e}-7$ \\
\hline 14 & $-8.230 \mathrm{e}-6$ & $-8.246 \mathrm{e}-6$ & $1.68 \mathrm{e}-8$ \\
\hline 15 & $-2.136 \mathrm{e}-7$ & $-1.722 \mathrm{e}-7$ & $-4.14 e-8$ \\
\hline 16 & $1.617 \mathrm{e}-6$ & $1.616 \mathrm{e}-6$ & $1.57 \mathrm{e}-9$ \\
\hline 17 & $-1.886 e-7$ & $-1.954 \mathrm{e}-7$ & $6.86 \mathrm{e}-9$ \\
\hline 18 & $-2.832 \mathrm{e}-7$ & $-2.819 \mathrm{e}-7$ & $-1.36 \mathrm{e}-9$ \\
\hline 19 & $9.175 \mathrm{e}-8$ & $9.269 e-8$ & $-9.46 \mathrm{e}-10$ \\
\hline 20 & $3.554 \mathrm{e}-8$ & $3.510 \mathrm{e}-8$ & $4.35 \mathrm{e}-10$ \\
\hline 21 & $-2.653 \mathrm{e}-8$ & $-2.659 \mathrm{e}-8$ & $6.87 \mathrm{e}-11$ \\
\hline 22 & $-7.936 \mathrm{e}-11$ & $1.543 \mathrm{e}+11$ & $-9,48 \mathrm{e}-11$ \\
\hline 23 & $5.251 \mathrm{e}-9$ & $5.238 \mathrm{e}-9$ & $1.31 \mathrm{e}-11$ \\
\hline 24 & $-1.557 \mathrm{e}-9$ & $-1.570 \mathrm{e}-9$ & $1.34 e-11$ \\
\hline 25 & $-5.445 \mathrm{e} \cdot 10$ & $-5.380 \mathrm{e}-10$ & $-6.45 e-12$ \\
\hline 26 & $4.906 \mathrm{e}-10$ & $4.909 \mathrm{e}-10$ & $-3.72 \mathrm{e}-13$ \\
\hline 27 & $-6.415 \mathrm{e}-11$ & $-6.546 \mathrm{e}-11$ & $1.31 e-12$ \\
\hline 28 & $-7.393 \mathrm{e}-11$ & $-7.354 \mathrm{e}-11$ & $-3.94 e-13$ \\
\hline 29 & $4.097 \mathrm{e}-11$ & $4.106 \mathrm{e}-11$ & $-9.72 e-14$ \\
\hline 30 & $-1.495 \mathrm{e}-12$ & $-1.605 \mathrm{e}-12$ & $1.10 e-13$ \\
\hline
\end{tabular}

The residue is the product of an $n$-independent factor with $\exp \left(i n \zeta_{p}\right)$, where $(n>0)$ and $\zeta_{p}$ is the pole in the upper half-plane. It follows that $\operatorname{Im}\left(\zeta_{p}\right)$ is the asymptotic rate of convergence, as defined earlier. It is easy to show that

$$
\mathfrak{I}\left(\zeta_{p}\right)= \begin{cases}\arcsin (b), & b \leqslant 1 \\ \pi / 2, & b>1\end{cases}
$$

Since the steepest terms are decreasing as $\exp (-n \pi / 4)$, it follows that for $b<0.707$, the residues dominate the asymptotic Fourier coefficients for sufficiently large $n$. For $b>2^{1 / 2} / 2$, i.e., when the poles are sufficiently far from the real axis, the rate of convergence is controlled by the steepest descent terms, and by them only, even though $u_{2}(z)$ has poles in the finite $\zeta$-plane. Figure 8 shows that for $b=1$, for example, the estimate $a_{n} \sim O(\exp [-n \pi / 2])$, based only on the poles of $u_{2}(z)$, is a wild overestimate of the actual convergence rate.

We can extend this argument to complex $b$ both graphically and analytically. The graphical approach is illustrated

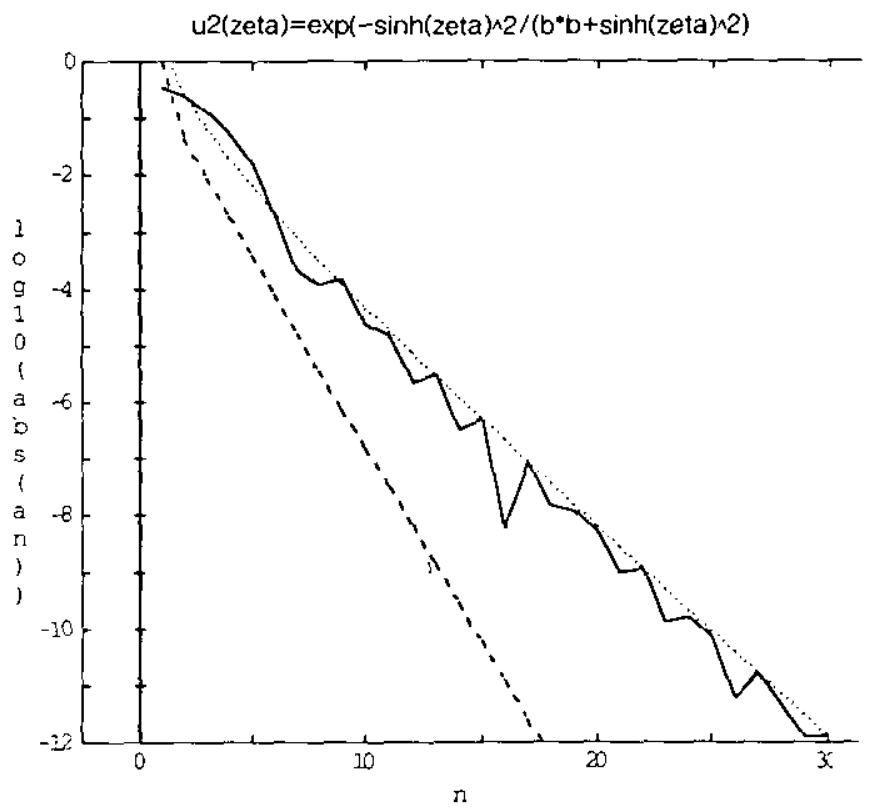

FIG. 8. Solid: $\log _{10}\left(\left|a_{n}\right|\right)$ of the Fourier coefficients of $u_{2}(z[\zeta])$, the Gaussian-with-poles for $b=1$. Dashed curve: Incorrect asymptotic approximation based on the residues-at-poles only, $a_{n} \sim$ [constant] $\exp (-n \pi / 2)$. Dotted: Correct asymptotic approximation based on the stationary point contributions only: $a_{n} \sim 3.72 n^{-3 / 2} \exp (-n \pi / 4)$.

in Fig. 4. The contours are lines of equal rates of convergence, that is, a pole on any particular contour line will produce the same asymptotic rate of convergence (for the contribution of that pole to the asymptotic Fourier coefficients) as a pole anywhere else on that contour. The heavy line is the contour $\mu=\pi / 4$, that is, the contour for which the asymptotic rate of convergence is equal to that of the stationary point terms for $u_{2}$. If the pole of $u_{2}(z[\zeta])$ (in the $z$-plane) lies above that contour, then the stationary point contribution will be exponentially large in comparison to the residue-at-the-pole term.

It follows that the conventional wisdom [1] that the rate of convergence is controlled by singularities of $u(z)$ at finite $z$ if any are present, is not always true. There is a precedent: Hille $[24,3,1]$ more than 50 years ago showed that a Hermite series would, under some circumstances, converge more slowly than predicted from its singularities.

Weideman and Cloot assumed that the conventional wisdom was true, as it is for their examples, and thus ignored the stationary point terms. However, this is not legitimate for all classes of functions. Fortunately, since both the stationary point contributions and the residues-atthe-poles give geometric convergence, their qualitative conclusion is correct, in general: When $L$ is allowed to grow logarithmically with $N$ so that the series truncation $E_{s}$ is roughly the same magnitude as the domain error, the total error decreases as $O(q N / \log (N))$. The possibility that the stationary point terms may be more important than the singularities is significant only in estimating the constant $q$. 
One can also explore the consequences of (5.11), i.e., that the asymptotic rate of convergence $\mu=\arcsin (b)$, by analytically exploring various limits. For example, if $u(z)$ has a singularity at $z=s+i z_{\mathrm{im}}$ where $s \Rightarrow \infty$ for fixed $z_{\mathrm{im}}$, then the asymptotic rate of convergence $\mu \approx z_{\mathrm{im}} / s$. However, the contribution from such a pole close to the real $z$-axis but far from the imaginary axis will be weighted by an exponentially small residue, assuming $u(z)$ decays exponentially on the line $\operatorname{Im}(z)=z_{\text {im }}$ as it does along the real axis. Simple experiments not shown here suggest that such singularities have little effect on the Fourier coefficients $a_{n}$ for reasonable $n$ for most functions.

\section{A GENERAL MODEL}

The steepest descent formalism can be applied with great generality. To illustrate this, consider

$$
u_{3}(\zeta)=\exp \left(\int^{\zeta} B\left(\zeta^{\prime}\right) \exp \left(r \zeta^{\prime \alpha}\right) d \zeta^{\prime}\right)
$$

where $B(\zeta)$ is arbitrary except that it varies more slowly than the exponential it multiplies and where $r$ and $d$ are constants. For simplicity, assume that $d$ is real. All the more specific models we have discussed and will discuss are special cases of this family of functions.

The stationary point condition for the asymptotic approximation of the Fourier integral of (5.12) is

$$
B\left(\zeta_{s}\right) \exp \left(r \zeta_{s}^{d}\right)=i n
$$

In the limit $\left|z_{s}\right| \Rightarrow \infty$ as $n \Rightarrow \infty$, this simplifies, as can be shown by taking the logarithm of (5.13), to approximately

$$
r \zeta_{s}^{d} \approx \log (n)
$$

or, equivalently,

$$
\zeta_{x} \sim \frac{\{\log (n)\}^{1 / d}}{r^{1 / d}}
$$

When $r$ is complex so that the function $u_{3}(\zeta)$ is a decaying oscillation as opposed to a monotonically decaying function, then (5.15) shows that the imaginary part of the stationary point is increasing logarithmically with $n$. This in turn implies supergeometric convergence for the stationary point contributions. If the function also has singularities in the finite $\zeta$-plane, then the singular terms will always dominate the asymptotic Fourier coefficients.

If, however, $r$ is real so that $u_{3}(\zeta)$ is decaying monotonically, at least for sufficiently large $|\zeta|$, then (5.15) gives only the real part of the stationary part, but says nothing about its all-important imaginary part. To obtain this, it suffices to apply Newton's iteration to (5.13), just once using (5.15) as the first guess. Discarding a small term, the logarithmic derivative of $\alpha$, we obtain

$$
\mathfrak{J}\left(\zeta_{s}\right)=\frac{\pi-2 \mathfrak{I}\left[\log \left(\beta\left(\zeta_{s}\right)\right) \mid\right.}{2 d r^{(2 d-1) / d} \log ^{(d-1) / d}(n)}
$$

where the argument of $\beta$ is evaluated at the first approximation to the stationary point given by (5.15).

Our first example, $u_{1}(z[\zeta])$, corresponds to the special case $d=1, r=2$ and $\operatorname{Im}(\log (\beta))=0$, i.e., $\operatorname{Im}\left(\zeta_{s}\right)=\pi / 4$. All functions of the form $\exp \left(-z^{p}\right)$ are mapped by the $\sinh$ transformation into $u_{3}$ with $d=1, r=p$. For this special case, the imaginary part of the stationary point is independent of $n$, implying geometric convergence. However, the rate of geometric convergence is controlled by the exponent $p$, i.e., $\mu=\pi /(2 p)$. Thus, a Gaussian has only half the asymptotic rate of convergence of an exponential whose argument is linear in $z$.

Our fourth example,

$$
u_{4}(z)=\operatorname{sech}^{m}(\lambda z) \Leftrightarrow u_{4}(\sinh (\zeta))=\operatorname{sech}^{m}(\lambda \sinh (\zeta))
$$

is also a special case of $u_{3}(z)$, one which asymptotes to a constant times $\exp (-m|z|)$. Equation (5.16) predicts that the steepest descent terms will decrease proportional to $\exp (-n \pi / 2)$.

The $m$ th power of the hyperbolic secant also has $(m+1)$ th-order poles at $z= \pm i \pi /(2 \lambda)$. For large $\lambda$, that is, very narrow peaks, the coefficients are asymptotically dominated by the contributions from the poles and decay as $\exp (-n \pi /(2 \lambda))$. When $\lambda \leqslant \pi / 2$, however, the singularities are sufficiently far up on the imaginary $z$-axis to lie on the $\mu=\pi / 2$ contour in Fig. 4 . The stationary point and residue terms are then proportional to the same exponential. However, the exponential for an $(m+1)$ th-order pole is multiplied by $n^{m}$, so the pole terms will dominate the Fourier coefficients by a factor of a power of $n$ for sufficiently large $m$, that is, when the hyperbolic secant function in $u(z)$ is raised to a sufficiently high power. (We have not attempted to determine the precise value of $m$ for which this occurs since this would require carefully determining the algebraic factor of $n$ which multiplies the exponential in the steepest descent term, as we did for our first two examples.)

\section{THE GAUSSIAN-OF-A-GAUSSIAN}

For the three previous specific examples, $u_{1}, u_{2}$, and $u_{4}$, the stationary point contributions decreased geometrically. One is tempted to conjecture that the stationary point terms always decrease geometrically, but this conjecture is false.

The sinh mapping always generates transformed functions in which the inner exponential is a linear function of $\zeta$ 
since $\sinh (\zeta)$ asymptotes to $\frac{1}{2} \operatorname{sgn}(\zeta) \exp (|\zeta|)$. This suggests that geometric or supergeometric convergence is the norm for functions obtained through the Weideman-Cloot mapping.

However, if we cut loose from the sinh mapping and consider more general entire functions, then it is easy to show that the rate of convergence need not be geometric. For example, the "Gaussian-of-a-Gaussian" function is

$$
u_{5}(\zeta)=\exp \left(-\zeta^{2} \exp \left(\zeta^{2}\right)\right)
$$

as illustrated in Fig. 9. This is a special case of $u_{3}(\zeta)$ with $d=2$. Eq. (5.16) shows that

$$
\begin{gathered}
\mathfrak{I}\left(\zeta_{s}\right) \sim \frac{\pi}{4 \sqrt{\log (n)}} \Leftrightarrow a_{n} \sim \gamma(n) \exp \left(-\frac{\pi n}{4 \sqrt{\log (n)}}\right), \\
n \Rightarrow \infty
\end{gathered}
$$

where $\gamma(n)$ denotes a factor that varies more slowly with $n$ than the leading exponential. Thus, the convergence is not geometric. However, it fails to be geometric only because of the logarithmic factor.

Figure 10 shows that the magnitudes of the Fourier coefficients are-at least until the $a_{n}$ are below the roundoff threshold of our 14 decimal point computationsindistinguishable from the straight line pattern which is the signature of geometric convergence on a log-linear plot. It is for this reason that we dubbed convergence like (5.19)-
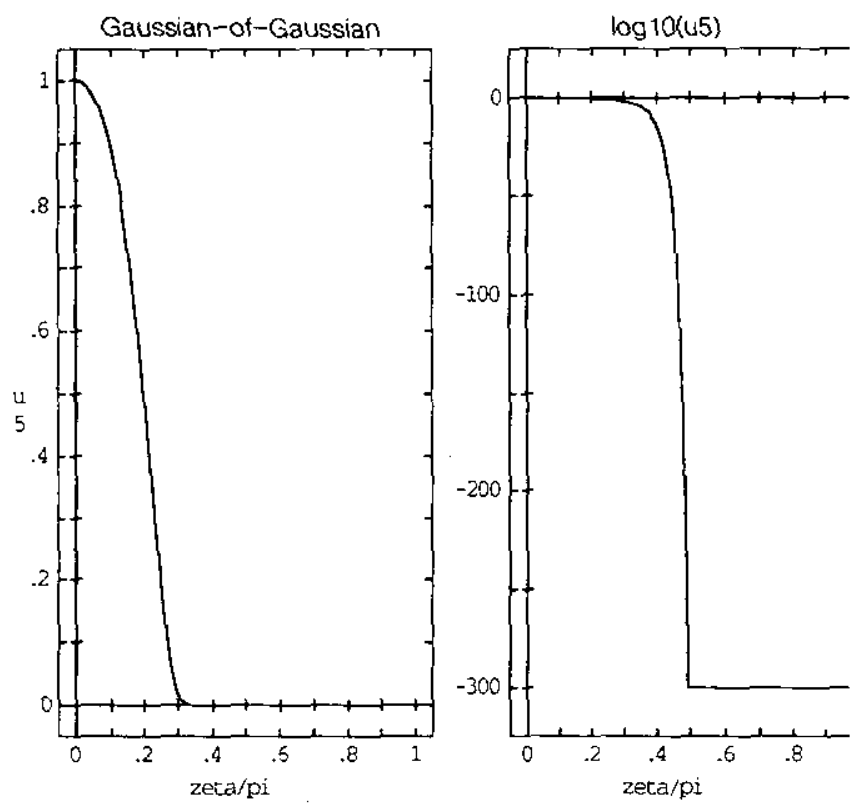

FIG. 9. Two views of the function $u_{5}(\zeta)=\exp \left(\zeta^{2} \exp \left(\zeta^{2}\right)\right)$. Only the half-interval $\zeta \in[0, \pi]$ is shown because the function is symmetric with respect to $\zeta=0$. The left panel uses a linear scale while the right graph shows the $\log _{\text {In }}\left(u_{5}(\zeta)\right)$.

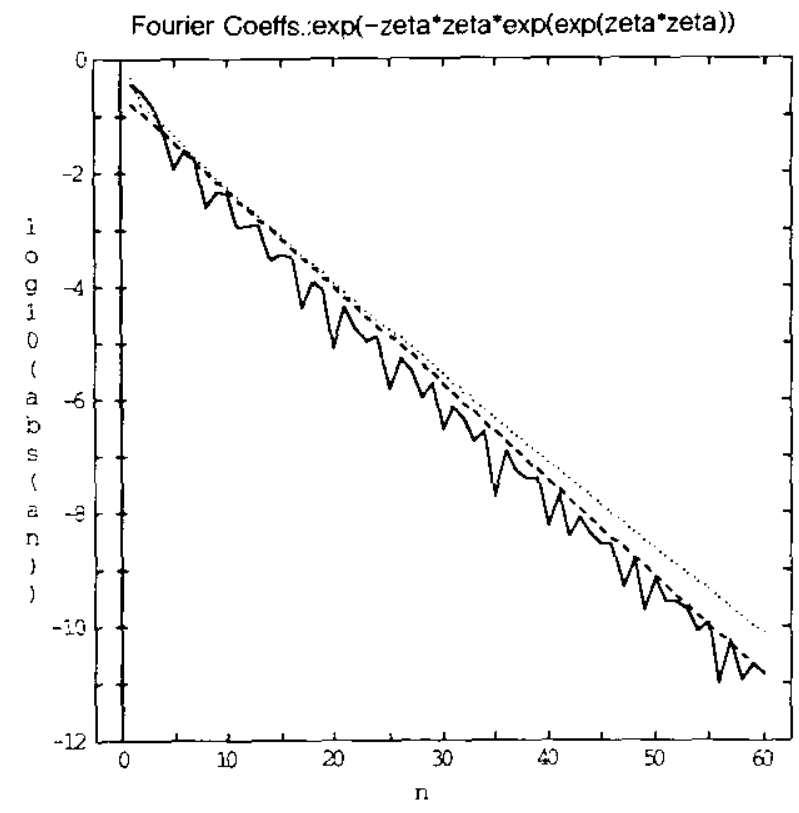

FIG. 10. Solid: $\log _{10}\left(\left|a_{n}\right|\right)$ for the Fourier coefficients of $u_{5}(\zeta)$, i.e., the imbricate series whose pattern function is the Gaussian-of-a-Gaussian. Dashed: $\log _{10}(\exp (-n \pi / 8)$ ). (The factor of $\pi / 8$ was chosen via empirical curve-fitting.) Dotted: same as the others but for $\exp \left(-n \pi /\left(4[\log (n)]^{1 / 2}\right)\right.$, the dominant factor in the asymptotic approximation.

geometric, except for a logarithmic factor inside the exponential-as "quasi-geometric." The dashed line in Fig. 10 is actually a much better empirical curve fit than (5.19) (with $\gamma$ taken as one for purposes of the graph).

The reason for this surprise is that $(5.19)$ is only an approximation. As shown in Table II, (5.19) is the first term in a power series in $v \equiv\{\log (n)\}^{1 / 2}$, which increases very, very slowly with $n$. For $n<60$, as shown in Fig. 11, the relative error in the location of the stationary points is never better than $10 \%$. The error is negligibly small only when $n$ is literally astronomical! Nevertheless, (5.19) is the correct lowest order asymptotic approximation.

\section{TABLE II}

Solutions of the Stationary Point Equation for the Imbrication of the Function $u_{2}(\zeta)=\exp \left(-\zeta^{2} \exp \left(\zeta^{2}\right)\right)$

\begin{tabular}{rcccr}
\multicolumn{1}{c}{$v$} & $n$ & $\zeta_{s}$ & \multicolumn{2}{c}{ Relative errors } \\
\hline 2 & 54.6 & $1.401+i 0.353$ & 0.107043 & -0.1128 \\
3 & 8103.1 & $2.369+i 0.267$ & 0.0218 & 0.0210 \\
4 & $8.886 \mathrm{e}+6$ & $3.404+i 0.206$ & -0.00530 & 0.0448 \\
5 & $7.200 \mathrm{e}+10$ & $4.450+i 0.164$ & 0.00140 & 0.0447 \\
6 & $4.311 \mathrm{e}+15$ & $5.494+i 0.136$ & 0.000314 & 0.0399 \\
7 & $1.907 \mathrm{e}+21$ & $6.532+i 0.116$ & -0.000149 & 0.0346 \\
8 & $6.235 \mathrm{e}+27$ & $7.565+i 0.101$ & -0.000110 & 0.0299 \\
9 & $1.506 \mathrm{e}+35$ & $8.594+i 0.0896$ & -0.000129 & 0.0259 \\
10 & $2.688 \mathrm{e}+43$ & $9.618+i 0.0804$ & -0.000122 & 0.0227 \\
\hline
\end{tabular}

Note. The errors were computed separately for the real and imaginary parts and $v \equiv\{\log (n)\}^{1 / 2}$. 


\section{THE DOMAIN OF CONVERGENCE OF THE FOURIER SERIES OF AN ENTIRE FUNCTION}

As noted earlier, the Fourier and Chebyshev series of entire functions often converge supergeometrically: This rate of convergence on the real axis implies convergence through the entire finite $\zeta$-plane. It has been assumed that this was always true for entire functions: supergeometric rate and an unbounded domain of convergence.

We have shown through steepest descents that this is not always true. The sinh mapping routinely generates functions whose stationary point terms are decreasing only geometrically. For the Gaussian, $u_{1}(z[\zeta])=\exp \left(-\sinh ^{2}(\zeta)\right)$; this implies that the series converges only within the domain $|\operatorname{Im}(\zeta)|<\pi / 4$ even though the function has no singularities except at infinity.

The Gaussian-of-a-Gaussian is even more extreme. The rate of convergence is subgeometric. This implies that the domain of convergence is the real axis only. This is as far as possible from the entire $\zeta$-plane!

\section{DOMAIN TRUNCATION ERROR AND VARIABLE MAP PARAMETER L}

In the analysis above, we have fixed $L$ and computed asymptotic approximations as $n \Rightarrow \infty$. These approximations can be interpreted in two ways: First, as approximations to the Fourier integral of a model function, and second, as approximations to the Fourier coefficients of the periodic functions formed by imbricating the model functions, as explained in Section 3. It is not correct to interpret these approximations as the total error in approximating a function on an infinite interval because the error in truncating the Fourier series after the $N$ th term must be augmented by the domain truncation error $E_{D}$, which is $O(|u(\zeta= \pm \pi)|)$.

In the limit $N \Rightarrow \infty$ for fixed $L$, the total error $E_{T}$ for the infinite interval problem tends not to zero but to $E_{D}$. To obtain convergence to zero, we must increase $L$ with $N$ so that the errors from truncating both the series and domain decrease simultaneously.

The optimum choice of $L$ is to balance the two errors so that they are roughly the same order of magnitude as explained in [2]. For geometrically converging series, it is a good approximation to take the last retained coefficient $a_{N}$ as the series truncation error $E_{S}[1,2]$. Then the optimum $L$ is obtained by solving

$$
\exp \left(-\frac{N \mu}{L}\right) \sim u(z=\sinh (L \pi))
$$

where the $L$-dependence follows from (5.1).
For example, for our first model function, the Gaussian, and for the Gaussian-with-pole when $b \gg 1$, the logarithm of both sides of (7.1) gives

$$
\frac{N \pi}{4 L} \sim \exp (2 \pi L)
$$

which implies that

$$
L \approx \frac{1}{2 \pi} \log (N \pi)+O(\log (\log (N)), \quad N \gg 1 .
$$

It follows that if we apply the Weideman-Cloot mapping to $u_{1}(z)=\exp \left(-z^{2}\right)$, the best we can obtain is a total error decaying roughly as

$$
E_{S}(N)+E_{D}(L(N)) \sim O\left(\exp \left[-\frac{\pi^{2} N}{2 \log (N \pi)}\right]\right) .
$$

The estimate is the same for the Gaussian-with-pole, as long as $b>1$. Weideman and Cloot [6] derived a similar estimate.

The presence or absence of the poles is much more significant for rival strategies for infinite interval problems. Boyd [2] shows that with his optimal algebraic mapping, one can obtain a total error for the Gaussian, $u_{1}(z)=\exp \left(-z^{2}\right)$, which decreases geometrically with $N$ :

$$
E_{S}(N) \sim O(\exp (-0.438 N))
$$

Because of the logarithmic factor in (7.4), the convergence for the Weideman-Cloot mapping is geometric, and therefore must be asymptotically inferior to Boyd's algebraic mapping.

However, the series coefficients for $\exp \left(-\sinh ^{2}(L \zeta)\right)$ decrease as fast as $O(\exp [-N \pi /(4 L)])$; for $L \leqslant 1.411$, this is faster than (7.5). It follows that for moderate $L$ and $N$, the Weideman-Cloot mapping is better. Boyd $[1,25]$ shows it is very common that a method which is superior to another method in the asymptotic limit is inferior for moderate $N$. The "crossover" point is at $L=1.411$, where the rate of convergence of the series coefficients for the Weideman-Cloot treatment of the Gaussian matches (7.5). For this $L$, however, the domain truncation error is

$$
E_{D}=\exp \left(-\sinh ^{2}[1.411 \pi]\right)=\exp (-1770.5)
$$

In other words, we need to use a map parameter for the Weideman-Cloot scheme as large as 1.411 only when we need about 770 decimal places of accuracy and can afford to take $N$ large enough to reduce the series truncation error to this same magnitude-a bit over 4000 . For smaller $L$ and $N$, the Weideman-Cloot mapping is superior to Boyd's map; for $N>4000$, his mapping is better.

It is clear that for all practical applications, the Weideman-Cloot strategy is better than an algebraic map- 
ping at least for entire functions of order two like $\exp \left(-z^{2}\right)$. Weideman and Cloot [6] describe several other numerical experiments which show that their change-of-coordinate is very effective for representing many other kinds of exponentially decaying functions on the infinite interval.

The basic reason for the practical effectiveness of their algorithm is that it is never necessary to vary their map parameter $L$ except within a very narrow range. Figure 5 shows, for example, that $L=1$ implies a domain error of $O\left(10^{-58}\right)$. One rarely needs more accuracy than this, so $L=1$ is a practical upper bound on the map parameter. However, choosing $L=\frac{1}{2}$ gives a boundary error as large as 0.005 . Thus, for our first model function, one would always choose $L$ in range $\left[\frac{1}{2}, 1\right]$. Using an arbitrary value in so narrow a range instead of the optimum $L(N)$ will not drastically increase the number of Fourier terms $N$ that must be retained to reach a given error tolerance.

In contrast, as shown by Boyd [2-4,26], the optimum map parameter for algebraic mappings varies widely with $N$ and also with the location of the poles and branch points of $u(z)$, to the extent that one knows them.

For non-entire functions, Boyd $[1,2,4,26]$ shows that poles or branch points significantly reduce the convergence rate of methods that use algebraic mappings, reducing the logarithm of the optimal error to $N^{r}$, where $r<1$, typically $\frac{1}{2}$ or $\frac{2}{3}$. Thus, for functions singular off the real axis, the Weideman-Cloot mapping is superior in the asymptotic limit $N \rightarrow \infty$ as they themselves note.

For best results, it may be helpful to make the simple linear stretching

$$
z=M y
$$

before applying the sinh mapping $z=\sinh (L \zeta)$. For $u(y)=$ $\operatorname{sech}^{m}(\lambda y)$, for example, the Weideman-Cloot mapping is most efficient when $\lambda \sim O(1)$. We can guarantee this by applying (7.7) first with $M \sim O(1 / \lambda)$.

The Fourier coefficients decrease more slowly as $L$ increases, so it is desirable to use the smallest $L$ which gives an acceptable domain error. If the goal is $d$ digits of accuracy, then one may optimize $L$ by choosing it so that

$$
E_{D}\left(L_{\mathrm{opt}}\right) \equiv u\left(\sinh \left[L_{\mathrm{opt}} \pi\right]\right) \approx 10^{-d} .
$$

For $u(z)=\operatorname{sech}^{m}(\lambda z)$, for example, this gives

$$
L_{\mathrm{opt}} \approx \frac{1}{\pi} \operatorname{arcsinh}\left(\frac{d \log (10)}{m \lambda}\right) .
$$

By replacing $u(z)$ by its asymptotic form as $|z| \Rightarrow \infty$ and then taking the logarithm of both sides of (7.8), one may derive similar estimates for quite general $u(z)$.

\section{SUMMARY AND OPEN QUESTIONS}

We show through steepest descent analysis that unlike the series for entire functions of finite order, the Fourier expansions for entire functions of infinite order may have only geometric or subgeometric convergence. This implies that instead of converging throughout the entire complex $\zeta$-plane (excluding infinity), as true of the series for functions of finite order, the Fourier series for entire functions of infinite order may diverge outside of a strip centered on the real $\zeta$-axis, or perhaps everywhere off the real axis. Because Chebyshev series are the image of a Fourier cosine series under the nonsingular mapping $z=\arccos (t)$, it follows that these statements apply to Chebyshev polynomial series as well.

To put it another way, Boyd's assertion is false [1]: That a Fourier series will converge within the largest strip in the complex plane where $u(\zeta)$ is free of singularities. The Fourier series of our Gaussian-of-a-Gaussian function converges only on the real axis even though it is free of poles and branch points everywhere except at infinity.

Through this steepest descent analysis, we have also been able to shed some light on the effectiveness of the hyperbolic sine mapping introduced by Weideman and Cloot for mapping a decaying function on an infinite interval into a function that can be accurately approximated by a Fourier series. We find that with or without the presence of singularities in $u(z)$, we can obtain "quasi-geometric" convergence in the sense that the error, with optimal choice of map parameter $L$, has a logarithm proportional to $N / \log (N)$, where $\cos (N \zeta)$ is the highest Fourier term kept in the truncation.

We have also learned that in comparing methods, such as the Weideman-Cloot mapping with alternative mappings proposed by Boyd [2-4] and Grosch and Orszag [8], one must be very careful to do more than merely inspect the asymptotic limit of infinite $N$. For finite $N$-even for very large $N$-the asymptotically superior strategy may in fact be markedly inferior for all practical parameter ranges. This caution about a "crossover $N$," where competing methods exchange superiority is especially relevant when $\log (N)$ factors appear in the asymptotic error estimates.

We conclude after this more careful analysis that the sinh mapping is always superior to algebraic mappings for functions with singularities in the finite $z$-plane, and it is also superior for entire functions for all reasonable values of error tolerance and Fourier truncation $N$.

Parenthetically, note that some other interesting applications of the sinh mapping (without domain truncation but with Whittaker cardinal functions) are given in $[17$, pp. 87-89;27].

A number of intriguing open questions remain. First, is it true that the Fourier series of all periodic functions which are the exponentials of entire functions of first order converge geometrically? Second, do series of periodic entire functions which are exponentials of entire functions of higher than first order converge quasi-geometrically? Third, is it possible to systematically classify entire functions of 
infinite order so as to replace vague phrases like "exponentials of entire functions of first order" by more precise categories?

Lastly, note that we have not offered any rigorous proofs for our assertions. The close agreement between the actual Fourier coefficients and the asymptotic approximation to them, as documented in Table $I$, is certainly encouraging. However, numerical agreement falls short of a proof. Our analysis suggests a whole slew of conjectured theorems.

\section{ACKNOWLEDGMENTS}

This work was supported by the NSF through Grants OCE8812300, DMS8716766, ECS9012263, and OCE9119459, and by the Department of Energy through Grant KC070101.

\section{REFERENCES}

1. J. P. Boyd, Chebyshev \& Fourier Spectral Methods (Springer-Verlag, New York, 1989).

2. J. P. Boyd, J. Comput. Phys. 45, 43 (1982).

3. J. P. Boyd, J. Comput. Phys. 54, 382 (1984).

4. J. P. Boyd, J. Comput. Phys. 69, 112 (1987).

5. J. P. Boyd, J. Sci. Comput. 3, 109 (1988).

6. J. A. C. Weideman and A. Cloot, Comput. Methods Appl. Mech. Eng. 80, 467 (1990).

7. A. Cloot and J. A. C. Weideman, J. Comput. Phys. 102, 398 (1992).

8. C. E. Grosch and S. A. Orszag, J. Comput. Phys. 25, 273 (1977).
9. A. B. Cain, J. H. Ferziger, and W. C. Reynolds, J. Compur. Phys. 56, $272(1984)$.

10. C. I. Christov, SIAM J. Appl. Math. 42, 1337 (1982).

11. C. I. Christov and K. L. Bekyarov, SIAM J. Sci. Stat. Comput. 11, 631 (1990).

12. K. L. Bekyarov and C. I. Christov, Chaos, Solitons and Fractals 5, 423 (1991).

13. J. P. Boyd, J. Approx. Theory 61, 98 (1990).

14. J. A. C. Weideman, A rational eigenfunction method for computing the Hilbert transform on the real line, Math. Comp., in press.

15. J. A. C. Weideman, Numer. Math. 61, 409 (1992).

16. D. Funaro, Polynomial Approximations of Differential Equations. (Springer-Verlag, New York, 1992)

17. J. Lund and K. L. Bowers, 1992: Sinc Methods for Quadrature and Differential Equations (Society for Industrial and Applied Mathematics (SIAM), Philadelphia, 1992).

18. D. Elliott, Math. Comput. 18, 274 (1964).

19. D. Elliott, Math. Comput. 19, 234 (1965).

20. D. Elliott and G. Szekeres, Math. Comput. 19 (1965).

21. G. F. Miller, Jr., SIAM J. Numer. Anal. 3, 390 (1966).

22. D. Gottlieb and S. A. Orszag, Numerical Analysis of Spectral Methods: Theory and Applications (SIAM, Philadelphia, 1977).

23. J. P. Boyd, $A d r$. in Appl. Mech., Vol. 27, edited by T.-Y. Wu and J. W. Hutchinson (Academic Press, New York, 1989), p. 1.

24. E. Hille, Trans. Amer. Math. Soc. 80 (1940).

25. J. P. Boyd, Appl. Math. Comput. 29, 49 (1989).

26. J. P. Boyd, J. Comput. Phys. 70, 63 (1987).

27. F. Keinert, J. Approx. Theory 66, 44 (1991). 